\title{
Penile Agenesis
}

National Cancer Institute

\section{Source}

National Cancer Institute. Penile Agenesis. NCI Thesaurus. Code C99009.

An extremely rare congenital abnormality characterized by the complete absence of the penis. It may be associated with other genitourinary abnormalities. 